\title{
Miracle on Sixth Avenue: Information Externalities and Search
}

By Andrew Caplin, Columbia University

John Leahy, Harvard University

December 1993

Discussion Paper No. 681

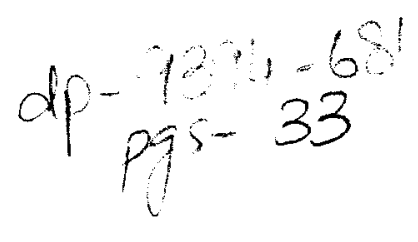




\title{
Miracle on Sixth Avenue: \\ INFORMATION EXTERNALITIES AND SEARCH
}

\author{
ANDRew Caplin and John Leahy \\ Columbia UnIVERStTy and HaRVARd University
}

We thank Charles Freeman for helpful comments, and we acknowledge research support from the National Science Foundation. 


\section{INTRODUCTION}

At one time Lower Sixth Avenue was one of the most fashionable retail centers in Manhattan, but the area collapsed as a retail center when the affluent moved further uptown and to the suburbs. Today, Sixth Avenue is in the midst of a rapid turnaround, as reported in the Sunday New York Times by Claudia Deutsch (June 27, 1993):

After decades of catering to virtually no one save the odd buyer of a quick lunch or piece of hardware, part of that once fashionable strip... is again turning into a major retail area.

How can we account for the revitalization of this depressed area in the middle of a recession?

The New York Times article provides an important clue. In 1992, Bed Bath \& Beyond opened Manhattan's first superstore in this area. It was the success of this store that prompted other stores to move to the area. One local businessman is quoted as stating that, "Sixth Avenue was dead as a doornail before, but Bed Bath \& Beyond has really opened it up," while another asserts that, "It was Bed Bath \& Beyond that really legitimized the neighborhood". The article goes on to point out that the arrival of the store changed local rents: "Bed Bath \& Beyond is said to be paying in the low $\$ 20$ 's a square foot. Barnes and Noble will be paying in the $\$ 30$ 's. Current negotiations in the area are inching towards $\$ 40$."

There are two features of the Sixth Avenue experience that we wish to explain. The first is the long period of under-use, and the second is the rapid rise in the area's prospects following the arrival and success of the first superstore. In this paper we develop a search model to explore one possible explanation for these events: information externalities. Before Bed Bath \& Beyond set up shop, all potential tenants faced the common problem of assessing whether or not New Yorkers would be willing to shop on lower Sixth Avenue which is a rather inconvenient location. The size of the crowds at Bed Bath \& Beyond, however, demonstrated to other retailers that many shoppers were indeed willing to make the trip. This knowledge led to the snowball effect as other tenants rushed to lease in the area.

We show that a simple search theoretic model with information spillovers can indeed explain both the long vacant period and the rise in rents following the success of Bed Bath \& Beyond. The basic idea is as follows. The vacant 
buildings on Sixth Avenue can be seen as options available for a wide variety of alternative uses. Committing a vacant unit to any one of its possible uses is costly to the owner, since such a commitment will normally involve modifying the unit to fit the needs of the particular tenant. If owners can learn about the best use for their property from the experience of neighboring properties, then they will have an incentive to delay renting. They will all play a waiting game, hoping that one of the other owners will be first to commit their property to a particular use and thereby generate the socially valuable information concerning whether or not the property is well matched to that use. Once a successful use is found the uncommitted owners will be able to use the information to improve the quality of their decisions.

One important feature of the model is that the delay until first occupancy is suboptimally long. This is due to the information externality. When deciding whether or not to accept a tenant, owners do not take into account the value to others of the information revealed in their match. Owners are therefore too selective in equilibrium. The model shows that the failure of optimality may be extreme. We provide examples in which social optimality demands immediate occupancy, but the market produces an arbitrarily long delay.

Another possible explanation for the sudden change in fortunes on Sixth Avenue is a crowd externality, in which later arrivals rushed in to take advantage of the crowds generated by Bed Bath \& Beyond. ${ }^{1}$ In this paper we also show how to incorporate crowd externalities a search model, and show that they differ in important ways from information externalities. In the crowd story the decisions of the various landlords are complementary; if one accepts a tenant, then this increase the size of the crowd and makes others more willing to accept tenants. In the information story the decisions of the various landlords are strategic substitutes; if one landlord is accepting a tenant then the others have an incentive to wait for the information revealed by this match. There is a big difference between being a leader when your mere presence encourages others to follow, and being a leader whose primary role is to warn others off making a similar mistake.

The model is suggestive of difficulties that may occur in many markets in which there are changes over time in the optimal pattern of usage of given resources, and in which there are costs of moving the resources between uses. We

1 The crowd externality story is implicit in many models of strategic complementarity, and is discussed in detail by Rauch [1993] in the context of shopping malls. 
believe that there are important analogies between vacancies in the property market and unemployment in the labor market. In both cases, a valuable resource is left unemployed for long periods of time, and in both cases we believe that there is an important role for information externalities. This line of thought is further developed in Caplin and Leahy [1993d].

To our knowledge, there are no other models of this form of information externality in a search market. ${ }^{2}$ The most closely related work is a line of research initiated by Zeira [1987] concerning the linkage between irreversibility of investment and the process of learning and information transmission (see also Rob [1991], Caplin and Leahy [1993a], Chamley and Gale [1992], and Caplin and Leahy [1993b]). Other related models that stress the underproduction of information in market equilibrium are due to Banerjee [1992], Bikhchandani, Hirshleifer and Welch [1992], and Vives [1992].

The paper is structured as follows. In Section 2, we present the basic search model. Section 3 demonstrates existence and uniqueness of the solution, and Section 4 explores the nature of this solution. In Section 5 we contrast the equilibrium outcome with the socially optimal outcome. In Section 6, we present extensions of the model to allow for recall of past offers, and to allow for different ownership structures. We also discuss the impact of other changes in the model, including the introduction of crowd externalities. Section 7 contains concluding remarks.

\section{THE MODEL}

Before the success of Bed Bath \& Beyond there appears to have been considerable skepticism concerning shoppers' willingness to return to Sixth Avenue. In the New York Times article, a local retail broker clearly states the grounds for this early uncertainty concerning shoppers' attitudes,

Let's face it, on a sunny afternoon Fifth Avenue is bustling and Sixth is deserted. Who knows to what extent bargain hunters will seek bargains that cost a $\$ 20$ round trip?

In this view, what Bed Bath \& Beyond did was to take a chance, betting that the traffic would turn out to be good. As the chief executive of Bed Bath \&

2 Externalities in the matching technology in search models are covered by Diamond [1981]. The externality that may be caused by division of the surplus in matching models is covered by Mortensen [1980]. 
Beyond put it, "Sure, we were worried about the lack of easy transportation and the low street traffic. But we wanted the Manhattan market...so we took a shot". When the store did indeed succeed, it revealed some important information about shoppers' behavior to other potential tenants who had been waiting in the wings. Other stores, such as Barnes and Noble, that had previously taken a wait and see attitude, observed the crowds at Bed Bath \& Beyond and decided themselves that Sixth Avenue was a good place to do business. ${ }^{3}$

To model this phenomenon we begin with a set of vacant buildings, each with distinct owners and several possible uses. For simplicity we assume that there are $N$ identical vacant buildings and that there are two potential uses for each property.

Owners must decide for which use their property is best suited. We use a search methodology to capture the time it takes for an owner to identify an appropriate tenant. Specifically, we assume that in each period, each vacant unit is visited by one and only one member of an ex ante homogeneous pool of potential tenants. All of these tenants come from the first of the two possible uses for the properties. This use corresponds to retail in the experience of Sixth Avenue. We will discuss the alternative use below.

We assume that the quality of a tenant is revealed to both the tenant and the owner of the unit during the visiting process. We denote the quality of a tenant by $\alpha$, which we take to be uniformly distributed over $[0,1]$. As detailed below, higher tenant types are able to make better use of the vacant unit and will therefore be able to pay higher rent. Once the tenant's type has been revealed to both landlord and tenant, the landlord decides whether or not to make the tenant a take it or leave it offer. Placing all of the market power in the landlord's hands removes the potential externality arising from a division of the surplus and allows us to focus on the effect of information spillovers. If the owner makes an offer then the tenant occupies the building. If the owner decides to wait then the owner meets a new potential tenant in the next period.

One essential ingredient of the model is a cost of transferring the buildings between uses, so that keeping a unit vacant is preferable to committing it to an arbitrary incorrect use. To capture the difficulty of transferring the building

3 In her article, Deutsch points out that Barnes and Noble had indeed backed out of a possible deal in 1991. 
between alternative uses, we make the extreme assumption that such transfers are impossible. Once a tenant agrees to move into a building, they pay the agreed to amount and occupy the property forever.

We assume that all tenants are risk neutral to simplify the landlord's problem of assessing the amount that each tenant is willing to pay for the unit. This means that landlords must work out the expected value of the payoff from occupancy to each tenant type. Uncertainty concerning these payoffs arises from the ex ante unknown market quality. This quality is captured by the parameter $\lambda$ which is drawn from a fixed distribution. $\lambda$ influences the amount that each vacant property is worth to all potential tenants from the first use. For simplicity we take it that $\lambda$ is uniformly distributed on $[0,2]$, and that the ex post payoff to a type $\alpha$ tenant in a type $\lambda$ market is the product $\alpha \lambda$. The market factor has an equiproportionate effect on the willingness to pay of all different $\alpha$ types, and is therefore a pure common factor. To further simplify the landlords' problem, we initially assume that there is no recall, so that landlords have only one chance to lease to each potential tenant. The case with recall is taken up in Section 6.

It is the parameter $\lambda$ that plays the key role in the information transmission process that corresponds to the uncertainty concerning shoppers' attitudes in the case of Sixth Avenue. The critical assumption is that information can only be released once a match is made; $\lambda$ remains unknown until after the first tenant occupies a building and that tenants success can be judged. Specifically, we assume that all participants in the market have access both to the terms of any occupancy agreement between any landlord and tenant, as well as the actual level of payoff that an occupied unit gives rise to. The observation of the amount the first tenant pays to take occupancy will reveal the tenant's $\alpha$ type, while the observation of the payoff will reveal the product $\alpha \lambda$ and therefore pin down the market type. Therefore in the period following the first match, $\lambda$ becomes public information, so that there is no risk left and an $\alpha$ type is willing to pay precisely $\alpha \lambda$.

The last critical assumption is that each vacant unit has fixed value $W>0$ in the alternative use. This assumption is needed to ensure that the information on $\lambda$ is relevant not only to the payoff, but also to the optimal decisions of the remaining tenants and landlords. We assume that at the beginning of each period, each owner either gets to accept a new searcher, or immediately and irrevocably accepts $W$ to put the vacant unit into its outside use. What this means is that if $\lambda$ turns out to be very low, all remaining units will be switched to the alternative use, 
while if it is high, the process of search will continue once $\lambda$ is revealed. In the case of Sixth Avenue, the alternative potential uses for the space include apartments (subject to zoning changes), storage, offices, and small scale retail outlets. In order for the model to be interesting it must be the case that $W$ is not so high that search is never worthwhile. A sufficient condition for this is introduced in Definition 2 in the next section. Finally, there is a discount factor $\delta \in(0,1)$ that motivates owners to let out their property sooner rather than later, other things equal.

Overall, the model accords with certain key features of the experience on Sixth Avenue. There is a common factor that can only be revealed by the completion of a match, just as the success of Bed Bath \& Beyond taught other potential retail tenants that crowds would show up. It is this ability to observe the success or failure of an occupied unit and to make inferences concerning the payoff to other units that is the distinctive feature of the model. The fact that the market factor is fully revealed by the first match is special. Fortunately the major conclusions will survive a great many changes in the microeconomic structure, provided only that there is more than one property owner, and there is some common information revealed after a match has been made.

As the above description makes clear, the informational assumptions in the model imply that the market passes through two phases. In the first phase before any units have occupants, there is no information on the market type, and the owners must simply decide on an acceptance strategy: which $\alpha$ types to permit to take occupancy for the payment of the amount $\alpha$ itself (the ex ante expected value to an $\alpha$ type given that $\lambda$ is uniform on [0,2] and the payoff is $\alpha \lambda$ ). The second phase begins in the period immediately after the first occupant has taken up residency, and involves a known value of $\lambda$. In this phase, there is a two fold decision facing the owners of vacant units. First, given the new information on $\lambda$, is it best to continue searching or to collect $W$ ? Second, if search is best, which $\alpha$ types should be permitted to take occupancy at $\operatorname{cost} \alpha \lambda$ (the ex post value to an $\alpha$-type)?

In looking to the model for an answer to these questions, we limit attention to equilibria in Markov strategies in which the decision rules of each agent depend only on information that is directly payoff relevant. In this case a strategy for an individual owner is a set of acceptable $\alpha$ types in the uninformed phase, a set of $\lambda$ values that warrant continued search through potential occupants, and a set of 
acceptable $\alpha$ types corresponding to those values of $\lambda$ that imply continued search. Given the information externality, the optimal strategies are interdependent, and we look for a symmetric Nash equilibrium.

The simple nature of the search framework leads to simple strategies. It is clear that any stationary equilibrium will involve cutoff rules: a reservation type $U$ in the uninformed phase, a cutoff level $\bar{\lambda}$, and a reservation type $I(\lambda)$ in the informed phase contingent on the realized value of $\lambda$. The Nash equilibrium condition is that all decisions must be optimal given that others are using the same decision rules. We summarize this equilibrium in Definition 1.

Definition: The data of the model are the number of vacant units $N \geq 2$, the outside use value $W$, and the discount factor $\delta \in(0,1)$. An equilibrium of the model consists a cutoff $\bar{\lambda} \in[0,2]$, a cutoff $U \in[0,1]$, and a function $I(\lambda):[\bar{\lambda}, 1] \rightarrow$ $[0,1]$ with the property that the following strategy is optimal for each landlord on the assumption that all others are pursuing it:

(a) In the uninformed phase with $\lambda$ unknown, the landlord accepts the occupancy of any tenant of type $\alpha>U$ upon payment of $\alpha$, and rejects potential tenants with $\alpha<U$.

(b) In the informed phase in which $\lambda$ is known, the landlord sells the property for $W$ if $\lambda \leq \bar{\lambda}$, and for values $\lambda \geq \bar{\lambda}$ the landlord allows search. When search is allowed, the landlord accepts the occupancy of any tenant of type $\alpha>I(\lambda)$ upon payment of $\alpha \lambda$ and rejects potential tenants with $\alpha<I(\lambda)$.

The existence and uniqueness of this equilibrium is demonstrated in Section 3, and a qualitative analysis is provided in Section 4.

\section{MODEL SOLUTION}

We apply standard dynamic programming logic to solve the model. We begin by solving for the optimal strategy of an informed landlord. Given that the actual value of $\lambda$ is known, an owner can mechanically compute the optimal reservation type $I(\lambda)$ using the known distribution of tenant valuations. Owners can then compute $\bar{\lambda}$ by comparing the value of this strategy to the value of the outside use. Finally, knowing their optimal decisions in the informed phase, owners can decide which offers to accept in the uninformed phase. This pins down $U$. 


\section{A. The Fully Informed Phase}

To solve for $I(\lambda)$, we initially ignore the presence of the outside use at value $W$. Let $V_{I}(\alpha, \lambda)$ denote the value of an optimal strategy for an informed owner who is visited by a potential tenant of type $\alpha$ and knows that the market type to be $\lambda$. By standard Bellman equation logic,

$$
V_{I}(\alpha, \lambda)=\max \left\{\alpha \lambda, \delta \int_{0}^{1} V_{I}\left(\alpha^{\prime}, \lambda\right) d \alpha^{\prime}\right\} .
$$

It is immediate from equation (3.1) that there exists a reservation level $I(\lambda)$ such that the optimal strategy is to accept the tenant if $\alpha$ is above this level and to reject if $\alpha$ is below. With this strategy the form of the value function is,

$$
V_{I}(\alpha, \lambda)= \begin{cases}\lambda . \alpha, & \text { if } \alpha \geq I(\lambda) \\ \lambda . I(\lambda), & \text { if } \alpha \leq I(\lambda)\end{cases}
$$

At $\alpha$ equal to $I(\lambda)$ the owner is indifferent between accepting the tenant and continued search. We can use this indifference along with equations (3.1) and (3.2) to solve for $I(\lambda)$,

$$
\lambda I(\lambda)=\delta \int V_{I}(\alpha, \lambda) d \alpha=\lambda \delta\left(\frac{1+I(\lambda)^{2}}{2}\right) .
$$

The first equality follows from indifference and equation (3.1). The second equality follows from equation (3.2). Equation (3.3) has a natural interpretation: the left hand side represents the value of accepting the marginal type $I(\lambda)$, while the right hand side is the expected value of rejecting this type in the hope that a higher type will show up.

It follows from equation (3.3) that $I(\lambda) \equiv I$ is independent of $\lambda$,

$$
\delta\left(1+I^{2}\right)=2 I .
$$

For any $\delta \in(0,1)$, it is clear that equation (3.4) has a unique solution for $I \in(0,1)$. To see this solve for $\delta$,

$$
\delta=\frac{2 I}{1+I^{2}}
$$

Now the right hand side of equation (3.5) increases monotonically from zero to one as $I$ increases from zero to one. Hence the optimal level of $I$ is continuous and increasing in $\delta$ and ranges over the entire open interval $(0,1)$. 
It will be convenient for what follows to treat the equilibrium level of $I$ rather than $\delta$ as a parameter of the model, leaving $\delta$ to be implicitly determined from equation (3.5). We shall take this perspective for the remainder of this section.

Given $I$, it is a trivial matter to add the outside option and decide whether or not an owner should continuing searching once $\lambda$ is known. The value of searching is $\int V_{I}(\alpha, \lambda) d \alpha=\lambda\left(\frac{1+I^{2}}{2}\right)$. This must be compared with $W$ to decide the optimal use. The property will be put over to the outside use if and only if $\lambda \leq \bar{\lambda}$ where

$$
\bar{\lambda}=\frac{2 W}{1+I^{2}} \text {. }
$$

With this the entire structure of the optimal decisions conditional on $\lambda$ being known is clear. This allows us to step back into the prior phase in which $\lambda$ is still unknown, and work out the optimal decision rule for these periods. Before doing this, we first make an assumption on $W$ that guarantees that the search problem is non-trivial, in the sense that $W$ is low enough to ensure that search is worthwhile in the uninformed state. It turns out that in order to ensure this, it is enough to assume that should the market turn out to take on its average quality, $\lambda=1$, then the owner would prefer to allow search in the informed stage (this assumption is sufficient but by no means necessary for initial search to be worthwhile, as we will see below). What this means is that,

$$
1 \geq \bar{\lambda}=\frac{2 W}{1+I^{2}} \equiv 2 w .
$$

Here we have introduced the transformation

$$
w \equiv \frac{W}{1+I^{2}} \in(0,1 / 2],
$$

to further simplify the derivation of optimal strategies in the uninformed phase. Note that the upper bound $w \leq 1 / 2$ would have been more complex to express in terms of the original data $W$ and $\delta$, since changes in $\delta$ change $I$ and therefore change the upper bound $W(\delta)$. This is one of the reasons that this transformation simplifies derivation of the solution to the model.

To summarize the analysis of the fully informed phase, we provide a listing of the transformed data of the problem and of the corresponding solutions for the equilibrium variables $I(\lambda)$ and $\bar{\lambda}$ :

DEFINITION 2: The transformed data of the problem are $N \geq 2, I \in(0,1)$ and $w \in(0,1 / 2]$, which give rise to unique equilibrium values $\bar{\lambda}=2 w$ and $I(\lambda)=I$ 
on $\lambda \in[\bar{\lambda}, 2]$. The corresponding values of the original data given in Definition 1 are uniquely given by $\delta=\frac{2 I}{1+I^{2}} \in(0,1)$ and $W=w\left(1+I^{2}\right)$.

\section{B. The Uninformed Phase}

Again we use dynamic programming logic to characterize the optimal decision rule in the uninformed period. We begin by ignoring the option of immediate sale for amount $w\left(1+I^{2}\right)$. We show below that with $w \leq 1 / 2$, this option is dominated by a strategy of search. We let $V_{U}(\alpha)$ denote the value of an optimal strategy for an owner who has a given searcher of type $\alpha$ in hand, but does not know the value of $\lambda$. The optimal decision is found by comparing the option of immediately filling the vacancy for the amount $\alpha$, the expected value of the property to the tenant given that $\lambda$ is unknown, with what happens if the offer is rejected. Here there are two possibilities. If no information is learned next period from the actions of others, then there is a repeat of the uninformed problem: we let $\operatorname{Pr}(\mathcal{U})$ denote the probability of this event, which will ultimately be determined in equilibrium. Otherwise, the actions of others reveal $\lambda$ by the next period. This event has probability $\operatorname{Pr}(\mathcal{I})=1-\operatorname{Pr}(\mathcal{U})$.

We can express that Bellman equation in the uninformed phase as follows,

$$
V_{U}(\alpha)=\max \left\{\alpha, \delta\left[\operatorname{Pr}(\mathcal{U}) \cdot \int V_{U}\left(\alpha^{\prime}\right) \cdot d \alpha^{\prime}+\operatorname{Pr}(\mathcal{I}) E V_{I}\right]\right\}
$$

where $E V_{I}$ denotes the ex ante expected value at the start of next period of being informed across the set of possible values of $\lambda$. From our analysis of the informed phase above, this expected value can be computed as,

$$
\begin{aligned}
E V_{I} & =\int\left\{\max \left[w\left(1+I^{2}\right), \lambda\left(\frac{1+I^{2}}{2}\right)\right]\right\} d \lambda \\
& =w^{2}\left(1+I^{2}\right)+\int_{2 w}^{2} \lambda\left(\frac{1+I^{2}}{2}\right) \frac{d \lambda}{2} .
\end{aligned}
$$

Expanding the integral yields,

$$
E V_{I}=\left(1+w^{2}\right)\left(\frac{1+I^{2}}{2}\right)
$$

The condition for indifference between continuation and acceptance that de- 
fines the reservation acceptance level $U$ is therefore,

$$
\begin{aligned}
U & =\delta\left[\operatorname{Pr}(\mathcal{U})\left(\frac{1+U^{2}}{2}\right)+\operatorname{Pr}(\mathcal{I})\left(\frac{1+I^{2}}{2}\right)\left(1+w^{2}\right)\right] \\
& =\operatorname{Pr}(\mathcal{U}) I\left(\frac{1+U^{2}}{1+I^{2}}\right)+\operatorname{Pr}(\mathcal{I}) I\left(1+w^{2}\right)
\end{aligned}
$$

Here the right hand side is a full specification of the expected return to turning down an offer of $U$ today: the first term is the discounted excess over $U$ provided no other tenant rents a property and the market remains uninformed, whereas the second term is the value if the information comes out with $E V_{I}$ expanded as in equation (3.10). The second equality follows from equation (3.5) which allows us to replace $\delta$ with a function of $I$.

To complete the characterization of $U$, we use the fact that this is a symmetric Nash equilibrium to substitute $\operatorname{Pr}(\mathcal{U})$. In a Nash equilibrium others are using the same cutoff rule to accept or reject tenants. The condition for remaining uninformed next period is that all other $N-1$ landlords received draws below $U$. This event has probability $\operatorname{Pr}(\mathcal{U})=U^{N-1}$. With this substitution, our final equation for $U$ is,

$$
U=U^{N-1} \cdot I\left(\frac{1+U^{2}}{1+I^{2}}\right)+\left(1-U^{N-1}\right) \cdot I .\left(1+w^{2}\right) .
$$

This completes the formal derivation of the model. We now establish existence and uniqueness of the equilibrium.

\section{Existence and Uniqueness of Equilibrium}

We show that the model possesses a unique equilibrium for any $I \in(0,1)$, $w \in(0,1 / 2]$, and $N \geq 2$ (corresponding to existence for any original data $N$, $\delta \in(0,1), W \in(0, W(\delta)])$.

Proposition 1: Consider any data $N \geq 2, I \in(0,1)$ and $w \in(0,1 / 2)$ of Definition 2. Corresponding to this, there exists a unique equilibrium of the form given in Definition 1 , with $I(\lambda)=I, \bar{\lambda}=2 w$, and $U \in\left(I, \min \left[1,\left(1+w^{2}\right) . I\right]\right)$ uniquely determined by equation (3.11). 
Proof: In order to establish existence and uniqueness of a solution we must show that for any given values of $I$ and $w$ in the appropriate ranges, equation (3.11) provides us with a unique solution for $U \in(0,1)$, which must have the additional property that it makes initial search more valuable than immediate sale. The condition for this is readily derived as,

$$
\frac{1+U^{2}}{2} \geq W=w\left(1+I^{2}\right)
$$

Since $w \leq 1 / 2$, this condition is satisfied provided $U \geq I$. Thus the demonstration of existence and uniqueness of equilibrium comes down to a proof that equation (3.11) has a unique solution $U \in(I, 1)$.

To prove this we rewrite the equation as,

$$
A_{1} \cdot U+A_{N-1} \cdot U^{N-1}=A_{0}+A_{N+1} \cdot U^{N+1} .
$$

where $A_{1}=1+I^{2}, A_{N-1}=\left[\left(1+w^{2}\right)\left(1+I^{2}\right)-1\right] I, A_{0}=\left(1+w^{2}\right) \cdot I .\left(1+I^{2}\right)$, and $A_{N+1}=I$. Note that $A_{0}>0$ so that the right hand side of (3.12) is strictly higher than the left hand side at $U=0$. Note also that $A_{1}+A_{N-1}>A_{0}+A_{N+1}$ so that at $U=1$, the left hand side is strictly higher than the right hand side. Since both sides are continuous, this establishes existence of a solution $U \in(0,1)$. Once we show that equation (3.12) has a unique solution, the fact that $U \geq I$ itself follows immediately from the observation that at $U=I$ the left hand side of (3.12) is still below the right hand side, and the fact that $U \leq\left(1+w^{2}\right)$.I follows from the fact that at $U=\left(1+w^{2}\right) . I$ the left hand side is above the right hand side.

The argument for uniqueness comes down to showing that the difference between the left and right hand sides in (3.12),

$$
D(U)=A_{1} \cdot U+A_{N-1} \cdot U^{N-1}-A_{0}-A_{N+1} \cdot U^{N+1},
$$

is quasiconcave in $U$ on the range $U \in[0,1]$, ruling out two distinct zeros since it starts below zero and ends above zero. To prove quasiconcavity, we show that at any point $\bar{U} \in[0,1]$ such that $D^{\prime}(\bar{U})=0$, the second derivative satisfies $D^{\prime \prime}(\bar{U})<$ 0 . Given that $D^{\prime}(\bar{U})=0$ we know,

$$
0=A_{1}+(N-1) A_{N-1} \cdot \bar{U}^{N-1}-(N+1) A_{N+1} \cdot \bar{U}^{N+1} .
$$

Differentiating again we find that,

$$
\begin{aligned}
D^{\prime \prime}(\bar{U}) & =(N-1)(N-2) A_{N-1} \cdot \bar{U}^{N-1}-(N+1) \cdot N \cdot A_{N+1} \cdot \bar{U}^{N+1} \\
& <\frac{(N-1) D^{\prime}(\bar{U})}{\bar{U}}=0 .
\end{aligned}
$$


The fact that the function is locally concave at any critical point implies that it can have only one such critical point, completing the proof of quasiconcavity. Q.E.D.

Note that in addition to providing the lower bound $U>I$, Proposition 1 demonstrates that there is an upper bound $\left(1+w^{2}\right) . I$. The presence of such a bound helps indicate the role of the outside value in the equation, and is also useful in the comparative static discussion in the next section.

\section{ANALYSIS OF MARKET EQUILIBRIUM}

Theorem 1 indicates that the basic model conforms to the experience in the Bed Bath \& Beyond case. In the uninformed phase each vacant lot has probability $1-U$ of accepting a tenant, and this event is independent across all lots. Thus there is probability $U^{N}$ that all lots remain unfilled in any uninformed period, and the expected time until the first unit is occupied is $\frac{1}{1-U^{N}}$. Once the information is out, the expected time until the next unit is occupied is $\frac{1}{1-I^{N}}$. The fact that in the case of Sixth Avenue it took longer to fill the first vacancy than subsequent vacancies has as its counterpart in the model the fact that $U>I$.

With regard to rental values, the model predicts an expected rent on the initial unit of $\frac{1+U^{2}}{2}$, while the expected rental on subsequent units should the match be sufficiently successful to keep the lots in the given use is,

$$
E\{\lambda \mid \lambda \geq 2 w\} \times \frac{1+I^{2}}{2}=\frac{(1+w)\left(1+I^{2}\right)}{2} .
$$

Theorem 1 demonstrates that $U \leq\left(1+w^{2}\right) . I$ so that,

$$
\frac{1+U^{2}}{2} \leq \frac{1+I^{2}\left(1+2 w^{2}+w^{4}\right)}{2} .
$$

The fact that $w \leq 0.5$ and $I \leq 1$ therefore ensures that the expected rental increases provided the match is successful enough to maintain the buildings in their original use. This was clearly the case in the setting of Sixth Avenue. Overall, the model predicts the extended initial period of vacancies, and both the higher rents and the increased speed with which lots were filled once Bed Bath \& Beyond succeeded.

The fundamental force that makes the model work is the information externality. Each owner must decide between letting out their property early and without the benefit of outside information, and instead waiting for the actions of 
another landlord-tenant pair to reveal the information to them. The possibility of waiting for another's actions to provide valuable information gives rise to a free rider problem. Landlords can only be induced to stop waiting if they believe that there is a sufficiently low probability that anyone else will accept an offer and hence reveal the information, and if they themselves have sufficiently attractive offers in hand. It is these forces that ensure that the equilibrium level of $U$ must be higher than it would be in the absence of the information externality. Once the information is out, there are no longer any interactions among the different vacant units, and all landlords conduct privately optimal searches among the remaining potential tenants.

The qualitative agreement between the model and the experience on Sixth Avenue does not give any hints as to the economic significance of the results. Among the questions to be answered are what happens in a large market? What happens as the outside option gets more valuable? In answering these questions, the main moral that can be derived from this section is that the delay till first occupancy can remain very long even as the number of vacant units increases without bound. This arises because of the potential severity of the free rider problems that discourages the release of information.

The basic comparative static propositions are summarized in Proposition 2.

Proposition 2: The equilibrium level of $U$ is strictly increasing in $w$, and is also strictly increasing in $N$.

Proof: The proof of the proposition is straightforward, and is based on analyzing equation (3.12). When $w$ increases with $N$ and $I$ fixed the only effect is to increase $A_{N-1}$ and $A_{0}$ by precisely the same algebraic amount,

$$
\Delta A_{N-1}=\Delta A_{0}=\left(\Delta w^{2}+2 w \cdot \Delta w\right) \cdot I \cdot\left(1+I^{2}\right)>0
$$

This means that if $w$ is increased and $U$ is left at the equilibrium value corresponding to the old value of $w$ then the left hand side will increase by $\Delta A_{N-1} \cdot U^{N-1}$ while the right hand side will increase by the larger amount $\Delta A_{0}$, so that the right hand side will exceed the left hand side at the old value of $U$. Finally, this shows that an increase in $W$ will call for an increase in $U$ to restore equality in equation (3.12).

In a similar manner, the only places in which $N$ shows up in equation (3.12) are in the exponents $U^{N-1}$ and $U^{N+1}$ respectively on the left and right hand sides 
of the equation. With $U \in(0,1)$, this means that an increase in $N$ with $U$ fixed at its original equilibrium value shrinks the second term of both the left and right hand sides by the same proportion. Therefore the left hand side shrinks more than the right hand side provided the term $A_{N-1} \cdot U^{N-1}$ exceeds the term $A_{N+1} \cdot U^{N+1}$ in the old equilibrium. This is equivalent to the statement that $A_{1} . U$ is less than $A_{0}$,

$$
\left(1+I^{2}\right) U \leq\left(1+w^{2}\right) \cdot I \cdot\left(1+I^{2}\right)
$$

or,

$$
U \leq\left(1+w^{2}\right) \cdot I
$$

which is true as stated in Theorem 1. This implies when $N$ is increased but $U$ is kept at the original equilibrium value, the left hand side of (3.12) falls below the right hand side, so that in increase in $U$ is required to restore equilibrium. Q.E.D.

These results are intuitive. It is natural to expect that an increase in the outside value will raise the payment needed to get an owner to commit to the risky use, since it raises $E V_{I}$, the amount that can be derived from just sitting and waiting for the information to be revealed. Similarly an increase in $N$ with $U$ fixed raises the probability that another agent would fill their vacancy this period would increase. This increases the value of rejecting a current offer and waiting for the information on the appropriate use of the property. Any force that increases the value of rejecting a current offer must by definition raise the minimum acceptable current offer, implying that $U$ itself must rise with $N$.

The fact that $U$ is increasing in $N$ does not imply that the expected waiting time till first occupancy, $\frac{1}{1-U^{N}}$, remains significant. One of the interesting issues is whether there are large markets in which there is still a significant delay till the first vacancy is filled. If $U$ were independent of $N$ then we know that adding enough vacancies would ultimately raise the probability of the information escaping in the first period to the limiting value of 1 . But it turns out that it is indeed possible for real delay to survive in the limit, as $U(N)$ rises toward 1 quickly enough to preserve a strictly positive probability of the vacancy remaining unfilled for many periods. The result is summarized in Proposition 3.

Proposition 3: The limiting value of $U(N)$ as $N$ increases depends on the value of $\left(1+w^{2}\right) . I$ :

(a) If $\left(1+w^{2}\right) . I \leq 1$, then $U(N) \rightarrow\left(1+w^{2}\right) \cdot I$, and $U(N)^{N} \rightarrow 0$. 
(b) If $\left(1+w^{2}\right) . I>1$, then $U(N) \rightarrow 1$ and $U(N)^{N} \rightarrow \tilde{U}$, where

$$
\tilde{U}=\frac{A_{0}-A_{1}}{A_{N-1}-A_{N+1}}=\frac{\left[\left(1+w^{2}\right) I-1\right] \cdot\left(1+I^{2}\right)}{\left[\left(1+w^{2}\right)\left(1+I^{2}\right)-2\right] . I} \in(0,1) .
$$

Proof: With Proposition 2 we know that $U(N)$ is increasing in $N$, we know that there is a limit as $N$ increases, $\bar{U} \equiv \lim _{N \rightarrow \infty} U(N)$. If $\bar{U}<1$, then equation (3.12) shows that $\bar{U}$ will satisfy,

$$
\bar{U}=\frac{A_{0}}{A_{1}}=\left(1+w^{2}\right) \cdot I
$$

For this to be valid, it must be the case that $\left(1+w^{2}\right) . I<1$.

In the remainder of the state space, it must be that $\bar{U}=1$. In this case we know that as $N$ increases, so $U^{N-1} \rightarrow U^{N+1} \equiv U(N)$. Substitution in (3.12) and rearrangement then shows that $U(N)$ itself approaches a limit value, $\tilde{U} \equiv$ $\lim _{N \rightarrow \infty} U^{N}$

$$
\tilde{U}=\frac{A_{0}-A_{1}}{A_{N-1}-A_{N+1}}=\frac{\left[\left(1+w^{2}\right) I-1\right] \cdot\left(1+I^{2}\right)}{\left[\left(1+w^{2}\right)\left(1+I^{2}\right)-2\right] \cdot I}
$$

It is readily seen that this lies in the range $[0,1]$ if and only if $\left(1+w^{2}\right) . I \geq 1$. The case with $\left(1+w^{2}\right) \cdot I=1$ is a cutoff case, with $\lim _{N \rightarrow \infty} U=1$ but $\lim _{N \rightarrow \infty} U^{N}=0$. Q.E.D.

Proposition 3 shows how severe the free rider problem can become. Note that with $w$ at its maximal value of $1 / 2, \tilde{U}$ approaches 1 as $I$ increases to 1 . What this means is that one can find large markets in which the expected wait till first occupancy, which heads toward $\frac{1}{1-U}$ as $N$ increases, is arbitrarily long. In contrast, in the next section we show that as $N$ increases, the probability of immediate information release always heads towards 1 in the socially optimal plan.

There is a good economic reason for the importance of the product $\left(1+w^{2}\right) . I$ in determining the nature of the free rider problem in large markets. Equation (3.10) computes the expected value of a vacant property at the beginning of a period in which the information on $\lambda$ has just been revealed as $E V_{I}=(1+$ $\left.w^{2}\right)\left(\frac{1+I^{2}}{2}\right)$. Viewed from the previous period, the expected value of a vacant property should someone else's match reveal the true state is therefore,

$$
\delta E V_{I}=\left(1+w^{2}\right) \cdot I,
$$

where we use equation (3.5) to substitute for $\delta$. 
If $\delta . E V_{I}$ is above 1 , then each landlord would prefer to wait for someone else's actions to reveal the true state rather than accepting even the maximal possible offer in the uninformed state. There can be no equilibrium in which the information is practically guaranteed to come out, since this requires some landlord to accept an offer of no higher than one, while guaranteeing an expected payoff above one for anyone that turns down such an offer. This means that as the number of firms increases, the probability of rejection must rise toward 1 fast enough to maintain aggregate uncertainty concerning whether or not anyone else will accept an offer.

In the following two sections, we provide further analysis of the free rider problem. In the next section we characterize the socially optimal strategies and show that in this case the optimal delay is zero in large enough markets. In Section 6 we extend the model to allow for recall of past offers, and show that this makes the free rider problem even more extreme. The possibility of recall greatly reduces the sacrifice to turning down an offer, and therefore makes it all the harder to induce an information revealing acceptance.

\section{THE SOCIAL PLANNER'S PROBLEM}

In this section we show that a social planner subject to the same informational constraints as the private market will face a greater incentive to accept an offer in the first period. The reason is simply that the planner internalizes the informational externality that the private market ignores. Derivation of the social optimum is simplified by noting that there are no externalities that remain once the information on $\lambda$ has been released. The solution to the social planner's problem comes down to specifying optimal acceptance rules for the periods before $\lambda$ has been revealed.

We consider a social planner setting a reservation cutoff level in the uninformed period, $R$, that is the same for all units. The per unit expected surplus generated by such a strategy can be written as $W(R)$,

$$
W(R)=R\left[\left(1-R^{N-1}\right) \delta E V_{I}+R^{N-1} \delta . W(R)\right]+\frac{1-R^{2}}{2},
$$

which can be rearranged to,

$$
W(R)=\frac{2\left(R-R^{N}\right) \delta E V_{I}+\left(1-R^{2}\right)}{2\left(1-\delta . R^{N}\right)} .
$$


The first two terms record the fact that if the individual unit receives an offer below $R$, then it is currently worth $E V_{I}$ at the start of next period if the information is revealed next period, which has probability $1-R^{N-1}$, and otherwise it is worth $W(R)$ at the start of next period. The final term reflects the surplus that arises if an offer above $R$ is received. The social planner's goal is to pick the value $R=S$ that maximizes $W(R)$. We show that the social planner's solution is always below the level $U$ set in the market equilibrium. In addition we show that in contrast to the market solution, the social planner will always set a strategy that ensures that the probability that the information is released in the first period approaches 1 as the market increases in size.

Proposition 4: A social planner picking the level of the uninformed reservation type to maximize the level of expected surplus given in equation (5.1) will always pick a level $S<U$. In addition, as $N$ increases, the probability that the information will be released in the first period increases to 1 in the solution to the social planner's problem.

Proof: The first order condition for the social planner's problem is,

$$
S=\left(1-N S^{N-1}\right) \cdot \delta E V_{I}+N \cdot S^{N-1} \cdot \delta W(S) .
$$

In contrast, we can express the first order condition for a single agent's optimal choice in the private Nash equilibrium (the equation for the reservation wage) in the form,

$$
U=\left(1-U^{N-1}\right) \delta E V_{I}+U^{N-1} . \delta W(U) .
$$

The difference is that the social planner does not take the other values of the cutoff rules as given in their optimization problem. The solution for the Nash equilibrium is a value of $U$ that satisfies equation (5.3) with $W(U)$ as defined in equation (5.1). The solution for $S$ satisfies equation.(5.2) again with $W(U)$ defined as in equation (5.1).

We use equation (5.3) to show $S<U$. Note that at $U=0$ the right hand side is $\delta E V_{I}$ which exceeds the left hand side. At $U=1$, the right hand side is $W(1)=0$, which is below the left hand side. Proposition 1 shows that there is a unique equilibrium and thus a unique point of equality in (5.3). This means that we can show $S<U$ by showing that the right hand side is still above the left hand 
side at $S$. To see that this is indeed so, let $S$ solve the social planner's problem. The first order condition (5.2) can be rearranged to,

$$
S+\delta(N-1) \cdot S^{N-1}\left[E V_{I}-W(S)\right]=\left(1-S^{N-1}\right) \cdot \delta E V_{I}+S^{N-1} . \delta W(S)
$$

Note that $E V_{I}>W(S)$ since it is better to be informed than uninformed. This means that in terms of equation (5.3), the right hand side evaluated at $S$ exceeds the left hand side, so that $S<U$.

We now consider whether it is possible for $S^{N}$ to head to a limit other than zero as $N$ increases. This is clearly impossible if $\delta E V_{I}<1$, since the average welfare functional (5.1) is diminishing for cutoff levels above $\delta E V_{I}$. Suppose that $\delta E V_{I} \geq 1$ and that $S$ heads toward 1 so fast that $S^{N}$ has a subsequence heading to a limit $L>0$. In this case substitution in equation (5.1) shows that welfare per unit heads to the limit $\mathcal{L}$,

$$
\mathcal{L}=\frac{(1-L)}{(1-\delta . L)} . \delta E V_{I}
$$

Against this, consider the (suboptimal) strategy of fixing $S=1-a$ for a fixed small value of $a>0$. In this case the average welfare heads to the limit $\mathcal{L}(a)$ identified from (5.1) as,

$$
\mathcal{L}(a)=\frac{2(1-a) \delta E V_{I}+2 a-a^{2}}{2}>(1-a) . \delta E V_{I}
$$

With $a=1-\frac{(1-L)}{(1-\delta . L)} \in(0,1)$ the alternative strategy dominates, so that the limit probability $S^{N}$ is zero if optimal strategies are pursued. Q.E.D.

It is the case of a large market that reveals just how poorly the market may handle information externalities. In intuitive terms, it is clear that increasing the number of vacant units raises the social value of the information on $\lambda$, since it linearly increases the amount that is learned. In contrast, Proposition 3 shows that with $\left(1+w^{2}\right) . I>1$, the minimal offer acceptable in the market heads toward 1 at a rapid enough rate to maintain aggregate uncertainty concerning whether or not an offer will be accepted. The failure of optimality is dramatic in large markets.

\section{EXTENSIONS}

In this section we consider a number of extensions to the basic model. We begin by adding a formal analysis of recall to the basic search model, and show that 
this merely increases the power of the information externality to distort market outcomes. We then analyze the effects of changing the ownership structure. We also consider the qualitative impact of changing the information structure and the nature of the payoffs. Finally, we show how to introduce crowd effects into the model.

\section{A. Recall in the Search Market}

The behavior of Barnes and Noble indicates that the vacant property market on Sixth Avenue is more appropriately modeled as allowing for recall. Barnes and Noble decided against taking a lease in 1991, but changed its mind once it saw the crowds at Bed Bath \& Beyond. To allow for recall, we amend the model by assuming that in any period each landlord can both get a new offer, and also recall any previous offer. The effect that this has on the optimal strategy in the fully informed phase is straightforward. With recall, let $M$ be the maximum of the past offers received by an owner once $\lambda$ is revealed: that is the maximum of the $\alpha$ types that has visited a particular vacant location. If $M$ is below the fully informed reservation cutoff type $I$ then it is irrelevant to optimal decision making. Otherwise the optimal strategy will be to sell immediately for $W$ unless the option of taking either $M$ or the first type (if higher) dominates: that is unless $\lambda\left(\frac{1+M^{2}}{2}\right) \geq W$, or

$$
\lambda \geq \frac{2 W}{1+M^{2}} .
$$

We can use this to solve for the optimal strategy in the uninformed period.

In the uninformed period it is natural to look for some reservation type $R \geq U$ and to accept offers of above $R$ and reject offers below. Given our knowledge of the optimal strategy once the truth has been revealed, we can write the indifference condition between acceptance and rejection of an offer in the uninformed phase as,

$$
R=\delta R^{N-1}\left(\frac{1+R^{2}}{2}\right)+\delta\left(1-R^{N-1}\right)\left[\frac{W^{2}}{1+R^{2}}+\left(\frac{1+R^{2}}{2}\right) \int_{\frac{2 W}{1+R^{2}}}^{2} \lambda \frac{d \lambda}{2}\right]
$$

The right hand side of (6.2) records the expected value if the offer of $R$ is turned down while others use this cutoff rule. The first term on the right hand side represents the probability that no information is revealed by any of the others multiplied 
by the expected value of remaining uninformed next period, while the second represents the expected value if the information does become public multiplied by the probability of that event. Equation (6.2) can be simplified to,

$$
2 R=\delta\left(1+R^{2}\right)+\delta w\left(1-R^{N-1}\right)
$$

where we have introduced

$$
w \equiv \frac{W}{1+R^{2}} \leq 1 / 2
$$

It is convenient to treat $w \in(0,1 / 2)$ rather than $W$ as the datum. This can be justified by noting that the value of $R$ that solves (6.3) is increasing in $w$, so that there is a unique value of $W=w\left(1+R^{2}\right)$ that corresponds to any given value of $w, \delta$ and $N$.

Equation (6.3) can be analyzed in an almost identical manner to equation (3.11) for the reservation cutoff with no recall. It is straightforward to show that equation (6.3) has a unique solution $R \in(U, 1)$, and that there is a unique equilibrium in which in the uninformed phase, any landlord accepts a type $\alpha$ tenant if and only if $\alpha \geq R$. Once the information has been revealed, any landlord with maximal offer $M \geq I$ allows in one more searcher and accepts the maximum offer between the old and new searcher, provided (6.1) is valid. For a searcher with maximal offer below $I$, the optimal strategy once the information has been revealed is identical to the optimal strategy in the case without recall.

This solution is very similar to the solution to the model without recall. The main difference lies in the fact that recall makes the free rider problem even more severe. This is because it greatly reduces the pressure to accept a high current offer in the uninformed phase, since there is the option of getting the same offer back again in the following period. The most graphic illustration of the increased severity of the free rider problem is seen by examining the behavior of $R^{N}$ as $N$ increases. It is readily seen from an examination of equation (6.3) that the sequence of non-revelation probabilities $R^{N}$ heads to a limit $\tilde{R}$ if and only if $w \geq \frac{1-\delta}{\delta}$, and that the limit obeys,

$$
\tilde{R}=1-\frac{1-\delta}{\delta w}
$$

The reason for the role of the difference between $w$ and $\left(\frac{1-\delta}{\delta}\right)$ in determining the limit behavior of the probability of non-revelation is exactly analogous to the reasoning in the case without recall. The key question is whether an owner would 
prefer to accept an offer of 1 , or delay till next period with the offer in hand and be assured of learning the value of $\lambda$. Consider an individual with an offer of 1 in hand deciding whether to delay or not. If they believe that the information is sure to come out next period, then they will have a return of $\delta \cdot \max [W, \lambda]$ if they wait till next period, which has an expected value of $\delta\left(1+\frac{W^{2}}{4}\right)$. This exceeds 1 if $\delta \frac{W^{2}}{4}>1-\delta$, and in these cases there can be no equilibrium with immediate revelation. In such cases, we know that the value of $R$ heads to 1 , so that $\frac{W^{2}}{4}$ approaches $w$, and the condition for limit delay involves the comparison between $w$ and $\frac{1-\delta}{\delta}$ noted above.

A numerical example show how severe the free rider problem can be in the case with recall. Suppose that it takes one year for a tenant to arrive at terms with a landlord, open their store, and have others gauge its level of success. This means that the relevant period length is one year. Suppose also that the discount factor is $\delta=0.9$ so that the interest rate is roughly $10 \%$ per annum, and that $w \approx 0.5$ (corresponding to a relatively high value in the outside use). In this case the limit of equation (6.5) is valid, and with a large number of vacant stores, the probability that none of them will be filled in the first year is $\tilde{R}=7 / 9$. This means that the expected time until the information becomes public is 4.5 years, while social optimality would call for the information to be made public in the minimum possible time of 1 year.

\section{B. Ownership Structure}

An important class of extensions involves allowing for alternative ownership structures. To understand why this is so important, note that a monopoly owner of all vacancies would be able to radically improve the functioning of the market by looking at the entire set of offers for the different units and accepting the best one in order to both release the information early and reduce the amount of wastage associated with committing units to the incorrect use. In this sense the monopolist has an advantage even over the social planner considered in the last section, who had to take into account the informational constraints arising from the assumed lack of direct communication between competitive landlords.

To understand the monopolist's optimal strategy, note that once the information has been released, the monopolist would follow exactly the same strategy as the competitive market. The difference occurs in the uninformed phase. Provided at least one offer is accepted during the period, equation (4.3) provides us with 
the current expected value of any that are unfilled as $\delta . E V_{I}=\left(1+w^{2}\right) . I$ This makes it intuitive that any offers above this should be accepted. The only issue is whether the maximum offer should still be accepted, even if it is below this level? The answer is yes if and only if the maximal offer exceeds some lower bound $M$. The entire issue of computing the monopoly optimum reduces to the identification of $M$. We compute $M$ for the relatively simple case in which $\left(1+w^{2}\right) \cdot I>1$ so that only one offer is ever accepted.

In this case we can solve for the socially optimal cutoff rule by comparing the value of accepting $M$, which has expected value $M+(N-1)\left(1+w^{2}\right) . I$, with rejection which has current expected value of $E$, which can be computed using the fact that the distribution of the maximum is $G(H)=H^{N}$,

$$
E=\frac{\delta\left[\left(N^{2}-1\right)\left(1+w^{2}\right) \cdot I\left(1-M^{N}\right)+N \cdot\left(1-M^{N+1}\right)\right]}{(N+1)\left(1-\delta M^{N}\right)} .
$$

With equation (6.6), we know that the condition for $M \in(0,1)$ to be an optimal cutoff is that it give rise to indifference between acceptance and rejection given continued use of the rule,

$$
M+(N-1)\left(1+w^{2}\right) \cdot I=\frac{\delta\left[\left(N^{2}-1\right)\left(1+w^{2}\right) \cdot I\left(1-M^{N}\right)+N \cdot\left(1-M^{N+1}\right)\right]}{(N+1)\left(1-\delta M^{N}\right)} .
$$

Rearrangement yields the indifference condition,

$$
(N+1) \cdot M+\left(N^{2}-1\right)\left(1+w^{2}\right) \cdot I(1-\delta)=\delta N+\delta M^{N+1} .
$$

The only other possibility is that acceptance always dominates rejection even with an arbitrarily small offer in hand. The condition for this is,

$$
\left(N^{2}-1\right)\left(1+w^{2}\right) \cdot I(1-\delta) \geq \delta N,
$$

where inequality (6.8) arises from substituting $M=0$ in equation (6.7), and noting that the left hand side represents acceptance.

We can characterize the monopoly policy as setting $M=0$ if inequality (6.8) does hold and otherwise setting $M$ as the unique solution $M \in(0,1)$ to equation (6.7). With this characterization, it is straightforward to show that $M<I$ by noting that at the point $M=I$ the right hand side of (6.8) surely already exceeds the left hand side. The reason that $M<I$ is that the information externality implies that the monopolist has an additional reason to allow a match to take place early: it reveals valuable information. Because matches create information 
that is valuable to other searchers, the monopolist accepts a tenant more readily than they would if they owned a single unit.

The monopoly optimum also has very different comparative static properties from the competitive outcome. Consider the effects of an increase in the outside value $W$, with $\delta$ and $N$ fixed. In economic terms, this makes the information on $\lambda$ more valuable, since the outside use is more attractive. As we have seen, this makes competitive private agents less willing to accept an offer in the uninformed phase, since they are made to give up an increasingly valuable option. But for the monopolist, the increased value of all vacant units makes it more important to release information early so that the proper use can be found for the vacancies. This suggests that an increase in $W$ should lower $M$, and this is indeed the case. This is readily seen for the case $\left(1+w^{2}\right) . I>1$ analyzed above, since the only effect of an increase in $W$ is to increase the left hand side of (6.7) and therefore call for a reduction in the minimally acceptable offer.

It is not surprising that where information externalities are important, there are certain advantages to monopoly ownership. The shopping malls that have sprung up in so many places indicate that these incentives have not gone unnoticed in the market. It is common for mall owners to offer good rates to certain key stores, both to provide the stamp of approval for the economic potential of the mall, and also to attract the crowds for the other stores that may arrive later.

The case with competitive owners appears more likely to characterize areas that are long established and have seen multiple uses, such as Sixth Avenue. In complex urban settings, the nature of the correlations between payoffs at different locations changes over time, and so the relevant peer group also changes. This means that absent monopoly ownership of the entire city, it is very likely that any new developments will cut across units that have different owners. This does not mean that there will not be efforts at consolidation and take-over once the common interest is noticed, but there is no evidence that such a process can take place without itself involving the expenditure of considerable time and effort. There are historical forces that may make the various owners more than a little suspicious in the face of a takeover bid that they can trace to a competitor. However this may be, the model points to the importance of ownership structure in determining the speed with which large numbers of vacant spaces get filled up. 


\section{Information Technology and Payoff Structure}

There are several conceptually straightforward amendments to the model that would reduce the extent of the information externality without fundamentally changing the message. If the payoffs at the various locations were less than perfectly correlated, then there would be less to be gained by waiting for another store's results. If there was a smaller cost of switching the unit among its various uses, then the loss caused by an incorrect decision would fall, and this would also reduce the free rider problem.

There are other changes that may operate to worsen the problem. One of these is the additional externality introduced when there are crowd spillover effects. As we show in the next section, this also tends to give rise to a suboptimal delay in filling the first vacant unit. Another such change would involve endogenizing the extent of the search effort. In this case the presence of other searchers would slow down the privately optimal search speed. Information is a public good and would be underprovided by private agents. The reduction in search speed would add another force tending to delay information revelation, especially in large markets.

Another set of issues arise in considering changes in the information structure. There are many ways to change the information flow that all end up reducing the extent of the learning that takes place from a single observation of a store's success. One way to assure this is to make the reasonable assumption that observations of rents and profitability are highly noisy. One could also imagine changing the structure of information and the protocol in individual bargains in a manner that would impede the flow of information among agents. While these amendments should be expected to reduce the extent of the discontinuity in market outcomes, there is little reason to believe that this would change the fundamental form of the externality.

\section{Crowd Effects}

We now amend the model to allow for crowd spillover effects. To keep things simple, we get rid of the information spillover. To capture the crowd effects in the simplest possible manner, we assume that the payoff to an $\alpha$ type in any period is $\alpha(1-\delta)$ while there are no other units occupied, and rises to $\lambda . \alpha(1-\delta)$ per period as soon as there is a single other unit occupied, with $\lambda>1$. This means that one store alone does not draw the crowd, but that the presence of two stores draws 
in the whole crowd. In all other respects we retain an identical model structure, although there is no role for the outside option $W$.

In this framework just as in the model of information spillovers, there is a two phase market history. In the first phase before any units have occupants, there is uncertainty concerning the timing of arrivals in the market and when the external economy will be in place. In the second phase, once a vacant unit is occupied, any later arrivals know that they will benefit from the full crowd spillover. Again equilibrium is given by reservation types $C$ with none occupied and $I$ once one or more unit is occupied. The model is identical to the information model in the second phase, so that the second cutoff is the familiar value of $I$ identified in equation (3.5) above.

To calculate $C$ we use the condition for indifference between acceptance and rejection. Note that both of these values are endogenous to the model and depend on the equilibrium strategies themselves. To see how much to charge an $\alpha$ type with no other occupants in place, the landlord must compute the expected present value of occupancy for this $\alpha$ type: it will be above $\alpha$ but below $\alpha . \lambda$. The expected surplus to an $\alpha$ type who takes occupancy in a period with no prior occupants depends both on the probability that some other unit gets occupied in this period, and the probability that units get occupied in subsequent periods. Hence the value depends on both $C$ and $I$ : the value can be written as $\alpha . K(C)$, where a little algebra shows,

$$
K(C)=\lambda-(\lambda-1) C^{N-1}\left(\frac{1-\delta}{1-\delta I^{N-1}}\right) .
$$

Note that $K(0)=\lambda$.

To make the optimal decision, one must compare the value of accepting the $\alpha$ type to the value of rejecting. If the offer is rejected then the following period involves either a repeat of the case with zero occupants, in which case the expected value as of the start of next period is $K .\left(\frac{1+C^{2}}{2}\right)$. If another unit has been occupied the expected value at the start of the next period is $\lambda .\left(\frac{1+I^{2}}{2}\right)$. A little further manipulation yields the indifference condition as between accepting and rejecting the offer in the uninformed stage as,

$$
C . K(C)\left(1+I^{2}\right)=C^{N-1} I\left(1+C^{2}\right) K(C)+\left(1-C^{N-1}\right) \cdot I\left(1+I^{2}\right) \cdot \lambda .
$$

Equations (6.9) and (6.10) are the final equations to determine $C$ and $K(C)$. 
It is straightforward to establish that an equilibrium exists, and that any solutions satisfy $C>I$, but $K . C<\lambda . I$ and $K .\left(1+C^{2}\right)<\lambda .\left(1+I^{2}\right)$. This means that the crowd story fits the same qualitative aspects. But there is a big difference, in that the crowd effect is in many ways less strong. In economic terms the reason for this is that the cost of leading is far higher in the model of information externalities, in which entry may be a permanent mistake. One reflection of this difference between the information model and the crowd model is shown by considering what happens as the number of vacancies grows. In contrast to the information story, in the crowd story the problem tends to disappear in a large market.

Proposition 5: In large markets as $N$ heads to infinity, any sequence of equilibria $C(N)$ head toward $I$.

Proof: The result hinges on an analysis of equation (6.10). We first show that the sequence $C(N)^{N}$ heads to zero. Suppose that there is a convergent subsequence of the $C(N)^{N}$ that head to some limit $\bar{C}$ that is strictly above zero. In this case the left hand side of $(6.10)$ heads to $\lambda\left(1+I^{2}\right)$, while the right hand side heads to $\lambda I .\left[2 \bar{C}+(1-\bar{C})\left(1+I^{2}\right)\right]$. A little rearrangement shows that for this equality to hold requires,

$$
\left(1+I^{2}\right)=I(1+I) \cdot \bar{C},
$$

which is impossible since the right hand side is strictly below $I+I^{2}$.

Since $C(N)$ heads toward zero, equation (6.9) implies that $K(C(N))$ heads toward $\lambda$ as $N$ increases. Now consider a sequence $C(N)$ that converge to some limit $\tilde{C} \in[0,1]$. Substitution in (6.10) then yields,

$$
\tilde{C} \cdot \lambda\left(1+I^{2}\right)=I\left(1+I^{2}\right) \cdot \lambda,
$$

so that $\tilde{C}=I$ as claimed.

Q.E.D.

The reason that Proposition 5 is valid is that with crowd spillovers, the more you trust someone else to accept a tenant, the more you wish to accept a tenant yourself. The. acceptance decisions of the various landlords are complementary. In a large market, if all tenants of type $\alpha \geq I$ are allowed in even before there are any tenants in place, the probability that another space will be filled rises to 1. There is no need to wait, because one is almost guaranteed that enough stores will arrive to get a crowd in the very first period. The more general issue is that 
while a large number of players may help to resolve the crowd externality problem, large numbers play a less essential role in resolving informational problems. In the information model, the fact that another landlord is accepting a tenant encourages delay, so that the acceptance decisions of distinct landlords are strategic substitutes rather than strategic complements.

In addition to this theoretical issue, there are practical reasons for viewing the information channel as the dominant channel in the case of Sixth Avenue. As we detailed in Section 2 above, the New York Times article contains a variety of quotes that indicate the great uncertainty concerning whether or not Bed Bath \& Beyond would succeed, so that there seems to be little doubt but that important information was released by its apparent success. In addition, the stores that have decided to locate in the area are very different from one another, so that it appears unlikely that the direct crowd effects are large. It is not obvious that the same shoppers who buy at Bed Bath \& Beyond will at the same time buy books from Barnes and Noble.

\section{CONCLUSIONS}

We have developed a theory of search with information externalities, and have shown how this may help account for the regeneration of Sixth Avenue in the middle of a recession. In qualitative terms, the key assumption is that the first store that makes the costly decision to locate must do so without knowing the level of some market level risk, and that their act of locating will help to reveal this to those who have waited. The end result is an incentive for all to free ride off the decisions of someone more anxious to locate than themselves.

We feel that similar forces are at work in the many markets in which there are changes over time in the optimal pattern of usage of given resources, and in which there are costs of moving the resources between uses. This makes it important to explore the applicability of analogous frameworks in the labor market and the capital market as well as the property market. At an even broader level, the model points to the importance of learning from the actions of others in the process of search and adjustment. We have argued elsewhere that such patterns of learning are pervasive in many markets (see Caplin and Leahy [1993c]), and we are actively exploring these issues in a variety of different settings. 


\section{REFERENCES}

Banerjee, A.V."A Simple Model of Herd Behavior," Quarterly Journal of Economics, 107 (1992), 797-818.

Bikhchandani, S., D. Hirshleifer, and I. Welch, "A Theory of Fads, Fashions, Customs, and Cultural Change as Informational Cascades," Journal of Political Economy, 100 (1992), 992-1026.

Caplin, A., and J. Leahy, "Sectoral Shocks, Learning, and Aggregate Fluctuations," Review of Economic Studies, 60 (1993a), 777-794.

Caplin, A., and J. Leahy, "Business as Usual, Market Crashes and Wisdom After the Fact," American Economic Review, forthcoming (1993b).

Caplin, A., and J. Leahy, "The Economics of Adjustment," in Rod Cross, ed. The Natural Rate of Unemployment Twenty Five Years On, Cambridge, Cambridge University Press, forthcoming (1993c).

Caplin, A., and J. Leahy, "Mass Layoffs and the Natural Rate of Unemployment," mimeo, Columbia University (1993d).

Chamley, C., and D. Gale, "Information Revelation and Strategic Delay in a Model of Investment," Boston University Working Paper 10, (1992).

Deutsch, C., "From the Carriage Trade to the Category Killers," Sunday New York Times, Property Section, p.13, June 271993.

Diamond, P., "Mobility Costs, Frictional Unemployment, and Efficiency, Journal of Political Economy, 89 (1981), 798-812.

Mortensen, D., "The Matching Process as a Non-cooperative Bargaining Game," in J.McCall, ed., The Economics of Information and Uncertainty, Chicago: University of Chicago Press (1982).

Rauch, J., "Does History Matter Only When it Matters Little? The Case of CityIndustry Location," Quarterly Journal of Economics, (108) 1993, 843-67.

Rob, R., "Learning and Capacity Expansion under Demand Uncertainty," Review of Economic Studies, 58 (1991), 655-75.

Vives, X., "Learning from Others", mimeo., Universitat Autonoma de Barcelona (1992).

Zeira, J., "Investment as a Process of Search," Journal of Political Economy, 95 (1987), 204-210. 


\author{
1993-94 Discussion Paper Series \\ Department of Economics \\ Columbia University \\ 420 W. 118 St., Room 1022 \\ New York, N.Y., 10027 \\ Librarian: Angie Ng
}

The following papers are published in the 1993-94 Columbia University Discussion Paper series which runs from November 1 to October 31. Domestic orders for discussion papers are available for purchase at $\$ 5.00$ (U.S.) each and $\$ 140.00$ (U.S.) for the series. Foreign orders cost $\$ 8.00$ (U.S.) for individual paper and $\$ 185.00$ for the series. To order discussion papers, please send your check or money order payable to Department of Economics, Columbia University to the above address. Please be sure to include the series number for the paper when you place an order.

671. Investment in U.S. Education and Training

Jacob Mincer ( Nov. 1993)

672. Freer Trade and the Wages of the Unskilled: Is Marx Striking Again?

Jagdish Bhagwati and Vivek Dehejia

673. Employer Size and Labor Turnover

Todd Idson

674. Less Crime May Be Worse

Brendan O'Flaherty

675. Team Production Effects on Earnings

Todd Idson

676. Language, Employment. and Earnings in the United States:

Spanish-English Differentials from 1970 to 1990

David Bloom and Gilles Grenier

677. The Impact of Performance Incentives on Providing Job Training to the Poor: The Job Training to the Poor: The Job Training Partnership Act (JTPA)

Michael Cragg

678. The Demands to Reduce Domestic Diversity among Trading Nations Jagdish Bhagwati

679. Mass Layotfs and I'nemployment Andrew (aplin and John Leahy 
680. The Economics of Adjustment Andrew Caplin and John Leahy

681. Miracle on Sixth Avenue: Information Externalities and Search Andrew Caplin and John Leahy

682. Arbitrage. Gains from Trade and Scoial Diversity: A Unified Perspective on Resource Allocation Graciela Chichilnisky

683. Who should abate carbon emissions?

Graciela Chichilnisky. Geoffrey Heal

684. Believing in Multiple Equilibria

Graciela Chichilnisky

685. L.muted Arbitrage. Gains trom Trade and Arrow's Theorem (jraciela Chichilnisky

686. International Emission Permits: Equity and Efficiency Graciela Chichilnisky. (jeotfrey Heal and David Starrett 